\title{
Structure and magnetic properties of a multi-principal element Ni-Fe-Cr-Co-Zn-Mn alloy
}

\author{
A. J. Zaddach ${ }^{\text {a }}$ C. Niu ${ }^{\text {a }}$ A. A. Oni ${ }^{\mathrm{a}}$, M. Fan ${ }^{\mathrm{a}}$, J. M. LeBeau ${ }^{\mathrm{a}}$, D. L. Irving ${ }^{\mathrm{a}}$, C. C. Koch ${ }^{\mathrm{a}}$ \\ ${ }^{a}$ Department of Materials Science and Engineering, North Carolina State University, Raleigh, \\ NC 27695-7907, USA \\ *Corresponding author: cckoch@ncsu.edu
}

\begin{abstract}
A nanocrystalline alloy with a nominal composition of $\mathrm{Ni}_{20} \mathrm{Fe}_{20} \mathrm{Cr}_{20} \mathrm{Co}_{20} \mathrm{Zn}_{15} \mathrm{Mn}_{5}$ was produced by mechanical alloying and processed using annealing treatments between $450-600{ }^{\circ} \mathrm{C}$ for lengths from 0.5-4 hours. Analysis was conducted using x-ray diffraction, transmission electron microscopy, magnetometry, and first-principles calculations. Despite designing the alloy using empirical high-entropy alloy guidelines, it was found to precipitate numerous phases after annealing. These precipitates included a magnetic phase, $\alpha-\mathrm{FeCo}$, which, after the optimal heat treatment conditions of 1 hour at $500{ }^{\circ} \mathrm{C}$, resulted in an alloy with reasonably good hard magnetic properties. The effect of annealing temperature and time on the microstructure and magnetic properties are discussed, as well as the likely mechanisms that cause the microstructure development.
\end{abstract}

Keywords: A. nanocrystalline metals ; B. magnetic properties ; B. phase stability ; C. mechanical alloying and milling; E. ab-initio calculations, F. electron microscopy, transmission 


\section{Introduction}

Conventional alloys are generally based on a single principal element with additional elements added to affect specific properties. Even complex alloys such as Alnico[1] and Inconel[2] alloys contain no more than 2 elements in concentrations above $20 \%$. Over the past several years, significant attention has been given to alloys containing five or more elements in equal, or nearly equal atomic ratios [3]. Alloys of this type can be difficult to design, as phase equilibria data is often unavailable for high-concentration multi-component mixtures beyond binary systems and common ternaries. Most focus is on solid solution alloys, called "highentropy" alloys (HEAs) due to their high configurational entropy [4]. However, limiting to solid solutions also limits the potential properties and applications, as the controlled formation of secondary phases can result in properties difficult or impossible to achieve in a single-phase material. Many engineering metals used today contain multiple phases. Therefore, some research has also been conducted on multi-principal element alloys that may not meet the strict HEA definition but have interesting properties. Several alloys have been developed with agehardening capabilities[5,6] while others have been alloyed with non-metal elements such as boron to form phases that aid in wear-resistance and high-temperature strength [7].

To date there has been little research on the magnetic properties of HEAs or other multiprincipal element alloys. The single-phase $\mathrm{NiFeCrCo}$ alloy has been shown to be ferromagnetic, with properties dependent on short-range chemical ordering. However, the magnetic properties of the ball-milled alloy at room temperature are poor and when produced by casting it has a Curie temperature near $100 \mathrm{~K}$ [8]. A BiFeCoNiMn thin film was found to have hard magnetic behavior after annealing [9] and hard magnetic behavior has been observed in an $\mathrm{AlCoCrCuFeNi}$ alloy [10]. 
In this study, we describe a nanostructured $\mathrm{NiFeCrCoZnMn}$ alloy prepared by mechanical alloying. This alloy is based on the previously described NiFeCrCoMn system, which has been widely studied for its mechanical properties and tendency for forming solid solution alloys $[11,12]$. We characterize the magnetic properties and microstructure as a function of heat treatment time and temperature, and describe how the magnetic properties relate to the microstructure. Empirical HEA design guidelines predict the alloy to form a stable solid solution, but we show from experimental and first-principles computational methods that formation of a multiphase alloy is energetically preferred.

\section{Methods}

Alloy powders were prepared by mechanical alloying elemental powders in a SPEX 8000 mixer mill. Powders of at least $99 \%$ purity were loaded into a stainless steel vial in a high-purity argon atmosphere with approximately $0.7 \mathrm{wt} \%$ dodecane as a process control agent (PCA). A 10:1 ball-to-powder weight ratio was used, with stainless steel balls. The sample was milled at room temperature for 24 hours. Before any analysis, the sample powder was put in a vacuum chamber for 18 hours to evaporate any dodecane remaining on the surface.

Samples of $\sim 0.09$ grams were compacted into $3 \mathrm{~mm}$ diameter cylinders using cold uniaxial pressing. Samples were then annealed at $450,500,550$, and $600{ }^{\circ} \mathrm{C}$ for $0.5,1,2$, and 4 hours in an $\mathrm{Ar}-2 \% \mathrm{H}_{2}$ atmosphere. Hardness tests were conducted using a Buehler microhardness tester with a $100 \mathrm{~g}$ load.

$\mathrm{X}$-ray diffraction (XRD) analysis was conducted on the as-milled powders as well as the samples annealed at $500{ }^{\circ} \mathrm{C}$ for 1 and 4 hours using a Rigaku SmartLab diffractometer with $\mathrm{Cu}$ $\mathrm{K} \alpha$ radiation. Phase analysis was done using Pananalytical HighScore Plus software with the ICDD PDF-4+ database [13]. Further analysis for lattice parameter determination was done 
using the PM2K Whole Powder Pattern Modeling program [14]. Compositional analysis of the as-milled and 2 hour $/ 500{ }^{\circ} \mathrm{C}$ samples were done using a Hitachi S3200N scanning electron microscope (SEM) equipped with an Oxford energy dispersive x-ray spectroscopy (SEM/EDS) detector, using an accelerating voltage of $30 \mathrm{kV}$.

Magnetization versus field (M vs. H) curves were measured using a Quantum Design MPMS SQUID-VSM at $1.8 \mathrm{~K}$ and $300 \mathrm{~K}$ with an applied field between $\pm 20 \mathrm{kOe}$.

The 1 hour $/ 500{ }^{\circ} \mathrm{C}$ sample was prepared for transmission electron microscopy (TEM) sample using an FEI Quanta 3D FEG dual-beam focused ion beam (FIB)/SEM with the in-situ lift-out technique. Energy dispersive X-ray spectroscopy (STEM/EDS) analysis was performed using a probe-corrected FEI Titan G2 60-300 kV S/STEM equipped with an X-FEG source operated at $200 \mathrm{kV}$. Bruker Esprit was used for post-processing with a 3-pixel smoothing filter to reduce noise. Standardless quantification was performed for all EDS spectra using the Q-map function. All EDS maps were formed using X-ray K-lines.

First principles calculations were performed by the exact muffin-tin orbital method combined with coherent potential approximation (EMTO-CPA) [15]. The Perdew-BurkeErnzerhof version of generalized gradient approximation (GGA-PBE) of exchange-correlation functionals was used $[16,17]$. The Kohn-Sham equations were solved within the so-called softcore approximation, which let the code recalculate core states after each iteration. The Green's function was calculated for 16 complex energy points. The basis set of EMTO included s, p, d, and $\mathrm{f}$ states. A $13 \times 13 \times 13 \mathrm{k}$-point mesh was used; the total energy converged within 1 $\mathrm{meV} / \mathrm{atom}$. The screened impurity model parameter of 0.902 was applied for electrostatic correction to the single-site CPA. Equilibrium lattice parameters were obtained by fitting 
volume-energy data to the Vinet equation of state [18]. Similar calculations have proven to agree well with experiments $[8,11]$.

\section{Results and discussion}

\subsection{Alloy structure}

The NiFeCrCoZnMn alloy forms a single-phase fcc solid solution after ball milling as shown in Figure 1a. The nominal composition of the alloy is $\mathrm{Ni}_{20} \mathrm{Fe}_{20} \mathrm{Cr}_{20} \mathrm{Co}_{20} \mathrm{Zn}_{15} \mathrm{Mn}_{5}$. As the atomic radius of $\mathrm{Zn}$ is significantly larger than the other components, its concentration was reduced from the equiatomic condition and balanced with $\mathrm{Mn}$, to reduce the average atomic size difference. SEM/EDS measurements of the as-milled alloy showed a higher Fe concentration $(\sim 23 \mathrm{at} \%)$ and reduced $\mathrm{Ni}$ and Co content, as is common with ball milling using steel media. The formation of a solid solution is predicted by the empirical HEA solid solution formation rules [19-21].

$$
\Omega=\frac{T_{m} \Delta S_{m i x}}{\left|\Delta H_{m i x}\right|}
$$

$T_{m}$ is the weighted average melting point of the constant elements, $\Delta S_{m i x}$ is calculated for an ideal mixture, and $\Delta \mathrm{H}_{\text {mix }}$ is calculated from the binary systems.

$$
\delta=\sqrt{\sum_{i=1}^{N} c_{i}\left(1-\frac{r_{i}}{\bar{r}}\right)}
$$

$\mathrm{c}_{\mathrm{i}}$ and $\mathrm{r}_{\mathrm{i}}$ are the concentration and atomic radius, respectively, of element $i$ and $\bar{r}$ is the weighted average atomic radius. Alloys that have high values of $\Omega(>1.1)$ and low values of $\delta(<6.6 \%)$ are likely to form solid solutions [3]. For this alloy, $\Omega=7.18$ and $\delta=4.31 \%$. Additionally, the largest $\left|\Delta H_{\text {mix }}\right|$ value for the binary systems in the alloy using Miedema's model is only $9 \mathrm{~kJ}$ $\mathrm{mol}^{-1}[22]$. These values suggest that the system should form a stable solid solution. 
However, after annealing at $500{ }^{\circ} \mathrm{C}$ for 1 hour, numerous additional peaks can be seen in the XRD results, shown in Figure 1b. Due to the complex nature of the alloy and large numbers of the elements, STEM/EDS analysis was used in conjunction with conventional automated XRD phase analysis. The presence of various phases can be observed in the EDS maps presented in Figure 2. Using this data, the phases in the alloy can be identified based on XRD results. Table 1 shows the phases identified in the 1 hour $/ 500{ }^{\circ} \mathrm{C}$ sample, their lattice parameters, the lattice parameters of the phase reported in the PDF-4+ database [13], and the average composition of 12-21 particles from the EDS maps. Due to the high degree of peak overlap, quantitative XRD phase analysis was not attempted. The carbon in the carbide phase was likely incorporated into the powder during milling from the organic PCA. The PDF entries referred to are 04-004-6329 (NiZn), 04-003-5514 (FeCo), and 01-089-2724 $\left(\mathrm{Cr}_{23} \mathrm{C}_{6}\right)$. No significant change in overall composition was observed by SEM/EDS after 2 hours at $500{ }^{\circ} \mathrm{C}$.

While the annealing temperatures used in this work are all below the FeCo order-disorder transition temperature, due to the highly non-equilibrium starting material and substantial amount (17.6\%) of impurity atoms in the phase, we assume the $\mathrm{FeCo}$ is the disordered $\alpha$ phase. In practice, the $\alpha$ and $\alpha^{\prime}$ phases are difficult to distinguish experimentally and have similar magnetic moments [23].

After the 1 hour $/ 500{ }^{\circ} \mathrm{C}$ anneal, the lattice parameter of $\mathrm{FeCo}$ is larger than the reported value, which agrees with the quantitative EDS results that show an enrichment in Fe and an average of $5 \% \mathrm{Zn}$ in the $\mathrm{FeCo}$ particles. The lattice parameter of $\mathrm{Cr}_{23} \mathrm{C}_{6}$ is slightly smaller than expected, likely due to substitution of Fe for Cr. The largest difference is seen in NiZn, which has a cell volume $0.45 \%$ smaller than the reference with the $\mathbf{c}$ vector having the largest difference from its expected value. EDS measurements suggest that $\mathrm{Zn}$ is below the 
stoichiometric ratio, with Mn being the largest impurity. As $\mathrm{Zn}$ is in the center of the cell, substituting the much smaller Mn atom would substantially decrease the cell height. As Ni is the smallest metallic element in the system, any substitutional impurities in the Ni sites would increase the a vector, as is also observed. The lattice parameter of the solid solution phase decreases by $1.1 \%$ compared to the as-milled alloy due to the loss of $\mathrm{Zn}$ to the NiZn phase. For reference, the close-packed atomic radii reported by Pearson [24] are also provided in Table 2.

Comparing the XRD results from 1 hour and 4 hour $500^{\circ} \mathrm{C}$ treatments, the relative intensity of the peak near $44.6^{\circ}$ increases after longer annealing times compared to the peak at $43.5^{\circ}$. The former corresponds to the FeCo $\{110\}$ while the latter is a mixture of the fec solid solution $\{111\}$ and NiZn $\{101\}$ peaks. This indicates that a larger fraction of the solution is transforming to $\alpha$-FeCo given longer annealing time. The lattice parameter of the solution phase decreases only slightly however, suggesting that the NiZn precipitation occurs much faster.

After treatment temperatures at $450{ }^{\circ} \mathrm{C}$ and $600{ }^{\circ} \mathrm{C}$, only a small amount of $\alpha$-FeCo forms. After 2 hours, the lattice parameter of the solid solution phase decreases by $0.4 \%$ and $1.4 \%$ at $450{ }^{\circ} \mathrm{C}$ and $600{ }^{\circ} \mathrm{C}$, respectively, indicating that $\mathrm{NiZn}$ formation is aided by higher temperatures. Carbide phases did not form at $450{ }^{\circ} \mathrm{C}$.

Based on the Scherrer method, the grain size of the as-milled alloy is approximately 7 nm. From measurements of 25-50 grains of each phase in STEM/EDS maps of several regions, it can be seen that the material remains nanocrystalline despite the phase changes. Grain size statistics of the precipitate phases can be seen in Table 3 . The true average may be smaller, as extremely fine particles are difficult to distinguish from the matrix. Many of the particles are irregularly-shaped, so the horizontal and vertical axis of each particle was measured. These sizes 
are consistent with mechanical tests of the 2 hour $/ 500{ }^{\circ} \mathrm{C}$ sample, which was found to have a relatively high hardness of $7.9 \mathrm{GPa}$.

The rate of NiZn precipitation appears to be related to temperature and occurs relatively quickly. FeCo precipitation however, occurs primarily within a narrow temperature window and takes several hours to approach completion, even at the optimal temperature. However, from the Fe-Co binary phase diagram [23,25], the $\alpha$ or $\alpha^{\prime}$ phase should be the equilibrium phase at all temperatures used near the equiatomic composition. Unfortunately, few relevant ternary phase diagrams for this system are available. In addition, it is reasonably well established that $\mathrm{NiFeCrCo}$ and $\mathrm{NiFeCrCoMn}$ form stable fcc solid solutions [8,21,26,27]. A likely mechanism is that as NiZn forms, it removes Ni from the matrix, destabilizing the solid solution phase, allowing FeCo to form. At higher $\left(\geq 600{ }^{\circ} \mathrm{C}\right)$ temperatures, however, the entropic contribution to the free energy stabilizes the solid solution. If all the $\mathrm{Zn}$ forms NiZn with most of the Ni from the matrix, the remaining 5-element mixture will have a configurational entropy of $11.7 \mathrm{~J} \mathrm{~mol}^{-1} \mathrm{~K}^{-1}$, assuming ideal mixing. Thus, at $600{ }^{\circ} \mathrm{C}$, the entropy will reduce the Gibbs free energy of the mixture by $10.2 \mathrm{~kJ} \mathrm{~mol}^{-1}$.

This mechanism reasonably agrees with experimental observations. At $450{ }^{\circ} \mathrm{C}$, only a small amount of NiZn forms and the remaining solid solution remains relatively stable. At 500$550{ }^{\circ} \mathrm{C}$, NiZn forms rapidly and a significant amount of FeCo forms from the solid solution. At $600{ }^{\circ} \mathrm{C}$, NiZn also forms rapidly, but only a small amount of FeCo forms, likely during cooling through $500{ }^{\circ} \mathrm{C}$. First-principles calculations of the formation energies of the phases also support this mechanism. The enthalpy of mixing of the annealed multiphase alloy is $-1.04 \mathrm{eV} /$ atom, lower than that of the as-milled alloy $0.00 \mathrm{eV} /$ atom. The formation of the FeCo and NiZn phases 
is therefore energetically preferred. The enthalpy of mixing of FeCo is $-0.12 \mathrm{eV} /$ atom, and that of $\mathrm{NiZn}$ is $-0.22 \mathrm{eV} /$ atom, indicating a higher driving force for the formation of NiZn.

Additionally, the milled alloy is nanocrystalline with an extremely small grain size and high defect density. This creates a non-equilibrium condition with high strain and interfacial energy with many triple junctions, grain boundaries, and dislocations that can serve as nucleation sites for precipitates.

\subsection{Magnetic properties}

The as-milled material is paramagnetic at room temperature and ferromagnetic at cryogenic temperatures, similar to the base $\mathrm{NiFeCrCo}$ alloy, and exhibiting a similar gradual transition from the FM to PM state [8]. However, its saturation magnetization at $1.8 \mathrm{~K}$ is lower, $27 \mathrm{emu} / \mathrm{g}$ compared to $>50 \mathrm{emu} / \mathrm{g}$ for ball-milled NiFeCrCo. This is likely due to the reduced amount of ferromagnetic elements and addition of antiferromagnetic Mn.

Figure 3 shows a representative $300 \mathrm{~K}$ M vs. $\mathrm{H}$ curve for the alloy after the 1 hour $/ 500^{\circ} \mathrm{C}$ annealing treatment. Figure 4 shows the saturation magnetization $\left(M_{s}\right)$, remanence $\left(M_{r}\right)$, intrinsic coercivity $\left(\mathrm{H}_{\mathrm{ci}}\right)$, and maximum energy product $\left(\mathrm{BH}_{\max }\right)$ of the alloy as a function of annealing temperature and time. The hard magnetic properties are optimized near 1 hour at $500{ }^{\circ} \mathrm{C}$, while $\mathrm{M}_{\mathrm{S}}$ continues to increase with longer annealing times. This supports the conclusions of the microstructure analysis, that the FeCo phase continues to form from the paramagnetic fcc matrix with extended annealing times.

After annealing at $450{ }^{\circ} \mathrm{C}$ or $600{ }^{\circ} \mathrm{C}$, the alloy is almost entirely paramagnetic at room temperature, though a small hysteresis loop is still present. Coercivity is least affected by 
temperature, with little difference between the $500{ }^{\circ} \mathrm{C}$ and $550{ }^{\circ} \mathrm{C}$ treatments at each time increment and relatively high values after $450{ }^{\circ} \mathrm{C}$ treatments.

As other multiphase metallic alloys are improved by thermomagnetic treatments $[28,29]$, a single sample was annealed for 1 hour at $500{ }^{\circ} \mathrm{C}$ in a $3 \mathrm{~T}$ magnetic field. There was no significant difference in coercivity or remanence compared to the sample annealed at the same temperature and time without a field. Saturation magnetization was slightly smaller, likely explained by differences in heating and cooling rates between the two furnaces. This is likely due to the lower Co content than commercial Alnico alloys, in which at least $24 \%$ Co is necessary for thermomagnetic treatments to be effective [30].

Since the FeCo grains are small $(<50 \mathrm{~nm})$, irregularly shaped, and generally isolated from each other within the matrix and other phases, they may be acting as single-domain particles, which would result in increased coercive force. This is supported by the reduction in coercivity with longer annealing times. While the amount of $\mathrm{FeCo}$ increases, increasing $\mathrm{M}_{\mathrm{s}}$, this likely occurs by coarsening rather than additional precipitation, increasing the particle size above the single-domain threshold. First-principles modeling by Burkert et al. predicted that certain tetragonally-distorted FeCo structures have extremely high magnetic anisotropy [31], but there was no significant distortion observed in this material despite the impurity content.

First principles modeling was also used in this study to calculate the magnetic moment of the alloy at $0 \mathrm{~K}$. This was compared to magnetization measurements at $1.8 \mathrm{~K}$. A random solid solution (RSS) was used to simulate the as-milled alloy. This RSS has a predicted moment of $0.43 \mu_{\mathrm{B}} /$ atom. To simulate the experimentally annealed material, we make the assumption that three phases are present. The first contains Fe and Co and forms as a RSS on the bcc lattice. The second contains $\mathrm{Ni}$ and $\mathrm{Zn}$, which forms an ordered $\mathrm{L} 1_{0}$ alloy. The third contains the remaining 
elements as a RSS on the fcc lattice with composition of $\mathrm{Cr}_{61.1} \mathrm{Mn}_{12.3} \mathrm{Fe}_{13.8} \mathrm{Ni}_{12.9}$. The total magnetic moment for the annealed material is then weighted by molar fraction based on total alloy composition and is predicted to be $0.82 \mu_{\mathrm{B}} /$ atom. The spontaneous magnetization was determined from the $1.8 \mathrm{~K}$ magnetization vs. field curves by extrapolating the saturation region to zero field using a linear fit. The spontaneous magnetization of the as-milled alloy is 22.6 $\mathrm{emu} / \mathrm{g}$, and that of the $1 \mathrm{hour} / 500{ }^{\circ} \mathrm{C}$ sample is $50.6 \mathrm{emu} / \mathrm{g}$. This is a $124 \%$ relative increase in the magnetic moment, which is a slightly higher percent increase when compared with the computationally predicted $91 \%$ increase of the magnetic moment. This disagreement may be due, in part, to a number of factors. First, the annealed alloy is simulated with three phases in isolation and no collective magnetic interactions between the phases are accounted for in the simulations. Second, as has been shown for similar complex solid solution alloys, the magnetic moment can vary by large amounts as a result of relatively small amounts of short-range atomic ordering [8], making prediction difficult. The possibility of short range chemical ordering in the simulated as-milled alloy was not considered. Including this effect may reduce the moment of the as-milled material and improve the percent increase. Regardless, the simulation results agree with the experimental trend that phase separation supports a significant increase in the bulk magnetic moment.

Compared to the few published HEA-based permanent magnets, $\mathrm{NiFeCrCoZnMn}$ performs well. The coercivity is nearly double that of $\mathrm{AlCoCrCuFeNi}$ and the remanence ratio $\left(\mathrm{M}_{\mathrm{r}} / \mathrm{M}_{\mathrm{s}}\right)$ is nearly tripled. The coercivity reported here is lower than that of BiFeCoNiMn, but $\mathrm{NiFeCrCoZnMn} \mathrm{can} \mathrm{be} \mathrm{produced} \mathrm{in} \mathrm{bulk} \mathrm{forms.}$

The results presented in this work represent the first attempt at producing a $\mathrm{NiFeCrCoZnMn} \mathrm{alloy.} \mathrm{Future} \mathrm{work} \mathrm{may} \mathrm{be} \mathrm{able} \mathrm{to} \mathrm{obtain} \mathrm{better} \mathrm{permanent} \mathrm{magnetic} \mathrm{properties}$ 
through optimization of the composition and processing. This may be achievable by further reducing the stability of the matrix phase relative to FeCo, increasing the driving force for precipitation. In this work, the effect of the $\mathrm{Cr}_{23} \mathrm{C}_{6}$ particles was not considered; future research may examine their influence on the microstructure and magnetic properties. They have the smallest particle size of the precipitate phases so they may serve to stabilize the nanocrystalline grain size via Zener pinning.

\section{Conclusion}

A hard magnetic NiFeCrCoZnMn alloy was produced using mechanical alloying and annealing. The alloy was designed using high-entropy alloy principles, but the solid solution phase formed during milling was found to be unstable at elevated temperatures. A combination

of XRD, STEM/EDS, and first-principles modeling were used to characterize the microstructure and determine the likely phase transformation mechanisms. We confirmed the formation of $\beta_{1}$-NiZn, $\alpha-F e C o$, fcc solid solution matrix and $\mathrm{Cr}_{23} \mathrm{C}_{6}$ phases. Despite being substantially similar to previously developed $\mathrm{NiFeCrCoMn} \mathrm{high-entropy} \mathrm{alloys,} \mathrm{the} \mathrm{behavior} \mathrm{of} \mathrm{the} \mathrm{alloy} \mathrm{disagreed}$ with the empirical models commonly used to develop HEAs. This illustrates the need for more robust alloy development guidelines for multi-principal element alloys

After optimal processing conditions, the alloy has a $300 \mathrm{~K}$ intrinsic coercivity of $515 \mathrm{Oe}$, saturation magnetization of $50 \mathrm{emu} / \mathrm{g}$, and maximum energy product of $0.155 \mathrm{MGOe}$. While this is not competitive with current generations of commercial magnets, it compares favorably to other HEA-based magnets and optimization of the composition may be able to improve these properties. Additionally, this system may offer other benefits, such as its high mechanical hardness, simple processing technique, and rare earth-free composition. 


\section{Acknowledgements}

- The work reported in this manuscript was made possible through support from the National Science Foundation from the Metals and Metallic Nanostructures program under grant DMR-1104930.

- A.A.O. and J.M.L gratefully acknowledge the Air Force Office of Scientific Research (Grant No. FA9550-12-1-0456).

- D.L.I. and C.N. would like to acknowledge Levente Vitos for sharing his EMTO-CPA code, version 5.7, for work on this project.

- The authors acknowledge the use of the Analytical Instrumentation Facility (AIF) at North Carolina State University, which is supported by the State of North Carolina and the National Science Foundation.

\section{References}

[1] C.A. Julien, F.G. Jones, J. Appl. Phys. 36 (1965) 1173.

[2] M. Sundararaman, P. Mukhopadhyay, S. Banerjee, Metall. Trans. A 23 (1992) 2015.

[3] Y. Zhang, T.T. Zuo, Z. Tang, M.C. Gao, K.A. Dahmen, P.K. Liaw, Z.P. Lu, Prog. Mater. Sci. 61 (2014) 1.

[4] J.W. Yeh, S.K. Chen, S.J. Lin, J.Y. Gan, T.S. Chin, T.T. Shun, C.H. Tsau, S.Y. Chang, Adv. Eng. Mater. 6 (2004) 299.

[5] S. Mohanty, N.P. Gurao, K. Biswas, Mater. Sci. Eng. A 617 (2014) 211.

[6] T.-T. Shun, C.-H. Hung, C.-F. Lee, J. Alloys Compd. 495 (2010) 55.

[7] C.-Y. Hsu, J.-W. Yeh, S.-K. Chen, T.-T. Shun, Metall. Mater. Trans. A 35 (2004) 1465.

[8] C. Niu, A.J. Zaddach, A.A. Oni, X. Sang, J.W.H. III, J.M. LeBeau, C.C. Koch, D.L. Irving, Appl. Phys. Lett. 106 (2015) 161906.

[9] C.-Z. Yao, P. Zhang, M. Liu, G.-R. Li, J.-Q. Ye, P. Liu, Y.-X. Tong, Electrochim. Acta 53 (2008) 8359. 
[10] S. Singh, N. Wanderka, K. Kiefer, K. Siemensmeyer, J. Banhart, Ultramicroscopy 111 (2011) 619.

[11] A.J. Zaddach, C. Niu, C.C. Koch, D.L. Irving, JOM 65 (2013) 1780.

[12] B. Gludovatz, A. Hohenwarter, D. Catoor, E.H. Chang, E.P. George, R.O. Ritchie, Science 345 (2014) 1153.

[13] ICDD, PDF-4+ 2010 (Database), International Centre for Diffraction Data, Newtown Square, PA, 2010.

[14] M. Leoni, T. Confente, P. Scardi, Zeitschrift Für Krist. Suppl. 2006 (2006) 249.

[15] L. Vitos, Computational Quantum Mechanics for Materials Engineers: The EMTO Method and Applications, Springer-Verlag, London, 2007.

[16] J.P. Perdew, K. Burke, M. Ernzerhof, Phys. Rev. Lett. 77 (1996) 3865.

[17] J.P. Perdew, K. Burke, M. Ernzerhof, Phys. Rev. Lett. 78 (1997) 1396.

[18] M. Hebbache, M. Zemzemi, Phys. Rev. B 70 (2004) 224107.

[19] X. Yang, Y. Zhang, Mater. Chem. Phys. 132 (2012) 233.

[20] Y. Zhang, Y. Zhou, J. Lin, Adv. Eng. Mater. 10 (2008) 534.

[21] F. Otto, Y. Yang, H. Bei, E.P. George, Acta Mater. 61 (2013) 2628.

[22] A. Takeuchi, A. Inoue, Mater. Trans. 46 (2005) 2817.

[23] I. Ohnuma, H. Enoki, O. Ikeda, R. Kainuma, H. Ohtani, B. Sundman, K. Ishida, Acta Mater. 50 (2002) 379.

[24] W.B. Pearson, The Crystal Chemistry and Physics of Metals and Alloys, John Wiley and Sons, New York, 1972.

[25] H. Okamoto, J. Phase Equilibria Diffus. 29 (2008) 383.

[26] S.C. Middleburgh, D.M. King, G.R. Lumpkin, M. Cortie, L. Edwards, J. Alloys Compd. (2014).

[27] B. Cantor, I.T.H. Chang, P. Knight, A.J.B. Vincent, Mater. Sci. Eng. A 375-377 (2004) 213.

[28] S. Sugimoto, J. Honda, Y. Ohtani, M. Okada, M. Homma, IEEE Trans. Magn. 23 (1987) 3193. 
[29] E.G. Povolotskii, Y.M. Dovgalevskii, V.K. Baitina, Met. Sci. Heat Treat. 5 (1963) 631.

[30] M. Stanek, L. Wierzbicki, M. Leonowicz, Arch. Metall. Mater. 55 (2010) 571.

[31] T. Burkert, L. Nordström, O. Eriksson, O. Heinonen, Phys. Rev. Lett. 93 (2004) 027203. 


\section{Table captions}

Table 1: Phases identified in the 1 hour $/ 500{ }^{\circ} \mathrm{C}$ sample, their lattice parameters (and estimated standard deviation), published lattice parameters from the PDF-4+ database [13], and composition.

Table 2: Close-packed atomic radii of the elements [24].

Table 3: Grain size statistics of precipitate phases in 1 hour $/ 500{ }^{\circ} \mathrm{C}$ sample.

\section{Figure captions}

Figure 1: $\mathrm{XRD}$ pattern of (a) as-milled $\mathrm{NiFeCrCoZnMn}$ alloy and (b) alloy after 1 hour, $500{ }^{\circ} \mathrm{C}$ anneal with main peaks of each compound identified.

Figure 2: STEM/EDS maps showing the distribution and morphology of precipitate phases after 1 hour, $500{ }^{\circ} \mathrm{C}$ anneal.

Figure 3: Magnetization vs. field curve for 1 hour $/ 500{ }^{\circ} \mathrm{C}$ sample

Figure 4: Magnetic properties as a function of annealing temperature and time. a) Saturation magnetization, b) intrinsic coercivity, c) remanence, and d) maximum energy product. 


\begin{tabular}{|c|c|c|c|c|c|c|c|c|c|c|}
\hline Phase & Param. & Value (nm) & esd (nm) & Reference[13] (nm) & $\mathrm{Ni}($ at \%) & Fe (at \%) & $\mathrm{Cr}(\mathrm{at} \%)$ & Co $($ at $\%)$ & $\operatorname{Mn}($ at\%) & $\mathrm{Zn}($ at \%) \\
\hline fcc SS & $\mathrm{a}$ & 0.3589 & 0.0002 & & 12.3 & 27.1 & 24.5 & 25.1 & 4.1 & 7.0 \\
\hline$\alpha-\mathrm{FeCo}$ & $\mathrm{a}$ & 0.2864 & 0.0001 & 0.2855 & 5.6 & 44.8 & 4.7 & 37.6 & 2.0 & 5.3 \\
\hline$\beta_{1}-\mathrm{NiZn}$ & a & $\begin{array}{l}0.2775 \\
03134\end{array}$ & $\begin{array}{l}0.0002 \\
00004\end{array}$ & $\begin{array}{c}0.2756 \\
031927\end{array}$ & 46.8 & 3.8 & 2.6 & 3.5 & 5.5 & 37.8 \\
\hline $\mathrm{Cr}_{23} \mathrm{C}_{6}$ & $\mathrm{a}$ & 1.0629 & 0.0004 & 1.065 & 5.1 & 14.0 & 65.3 & 8.7 & 3.2 & 3.6 \\
\hline
\end{tabular}


Table 2

$\begin{array}{cc}\text { Atom } & \text { Radius }(\mathbf{n m}) \\ \mathrm{Ni} & 0.1246 \\ \mathrm{Fe} & 0.1274 \\ \mathrm{Cr} & 0.1282 \\ \mathrm{Co} & 0.1252 \\ \mathrm{Mn} & 0.1264 \\ \mathrm{Zn} & 0.1394\end{array}$




$\begin{array}{cccccc}\text { Phase } & \text { Mean }(\mathbf{n m}) & \text { Median }(\mathbf{n m}) & \text { SD }(\mathbf{n m}) & \text { Min }(\mathbf{n m}) & \text { Max }(\mathbf{n m}) \\ \mathrm{FeCo} & 29.7 & 27.3 & 12.6 & 10.7 & 86.6 \\ \mathrm{NiZn} & 41.8 & 35.6 & 24.4 & 14.4 & 135.1 \\ \mathrm{Cr}_{23} \mathrm{C}_{6} & 28.9 & 25.1 & 10.7 & 15.7 & 65.7\end{array}$




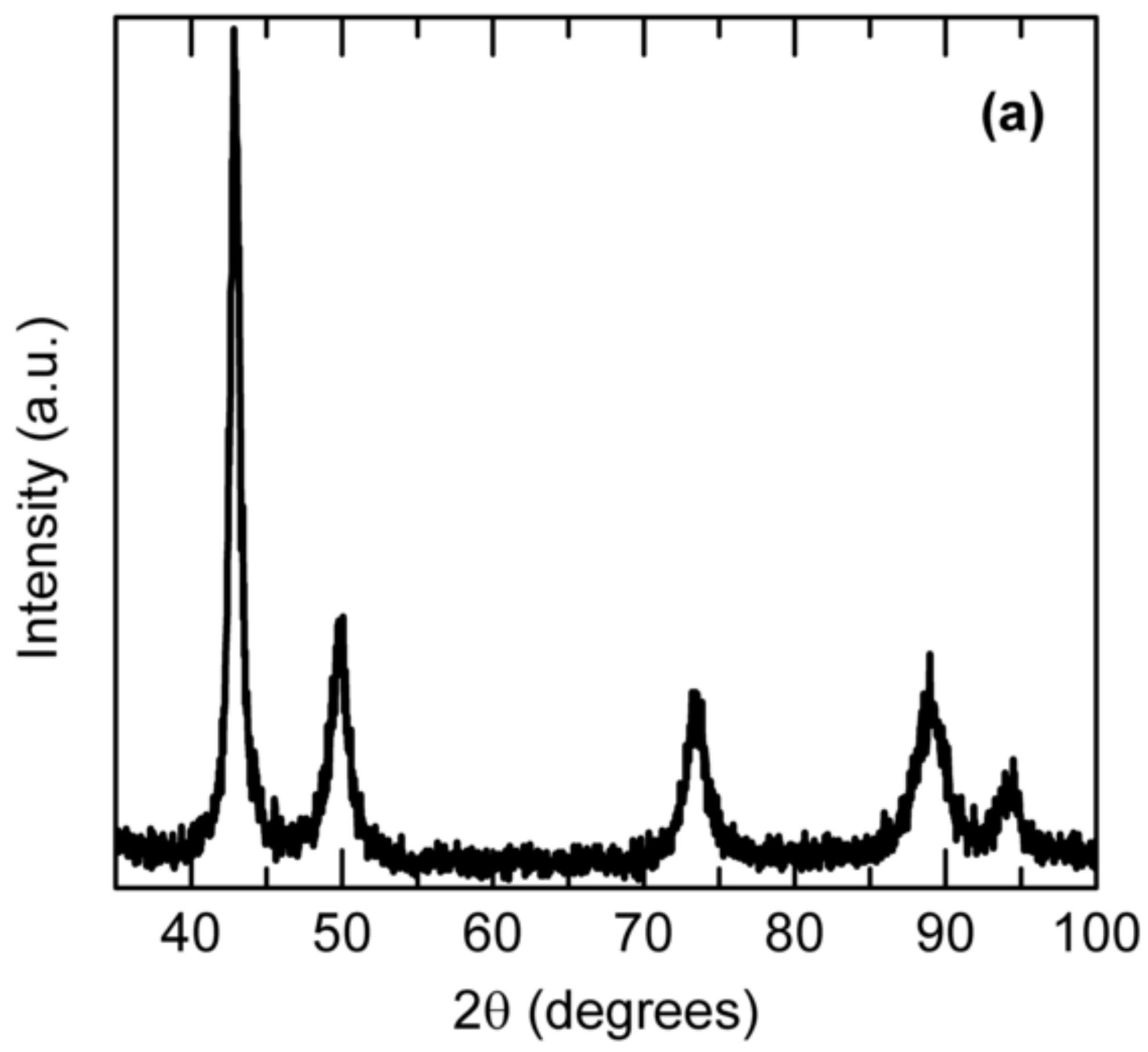




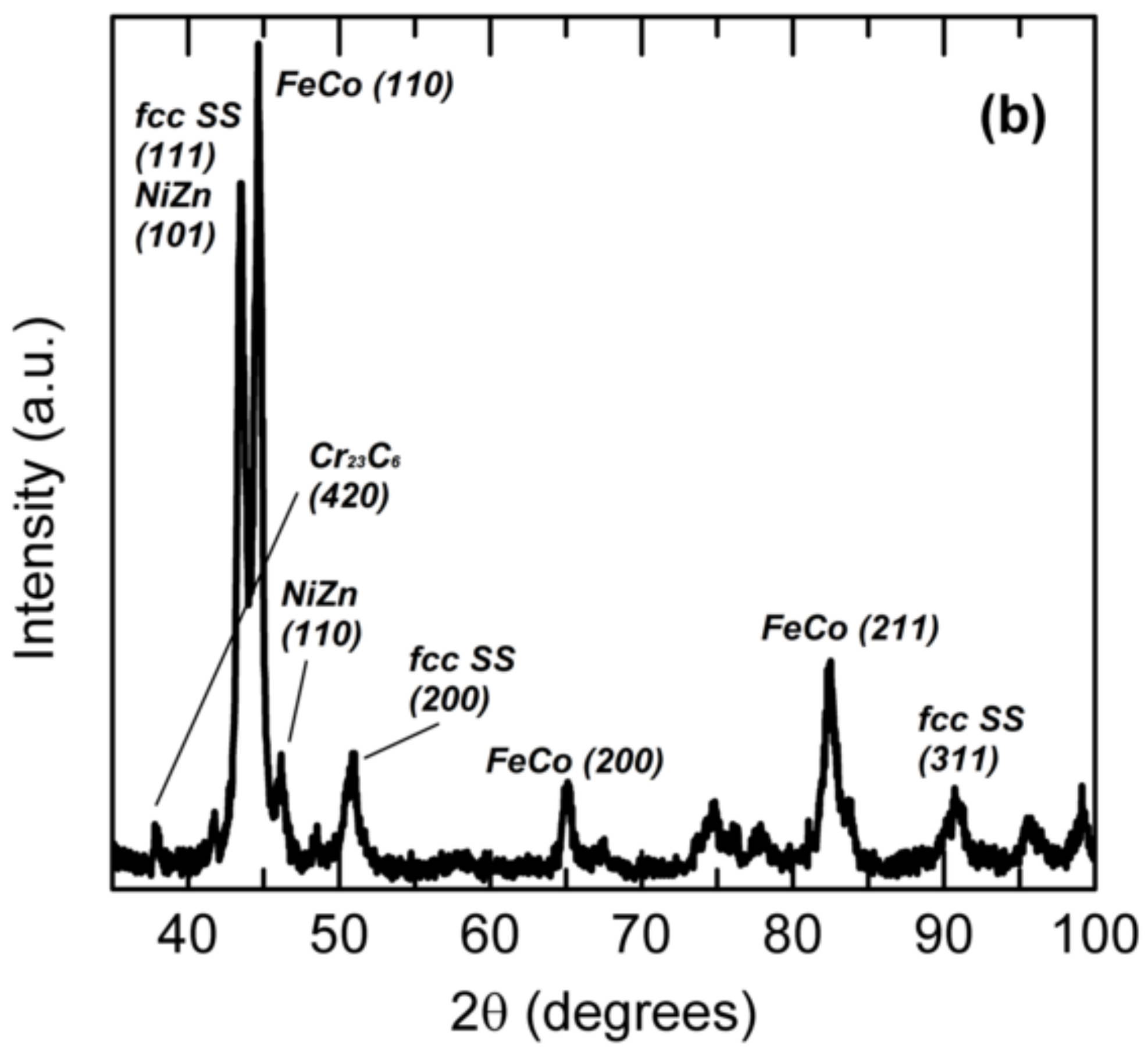



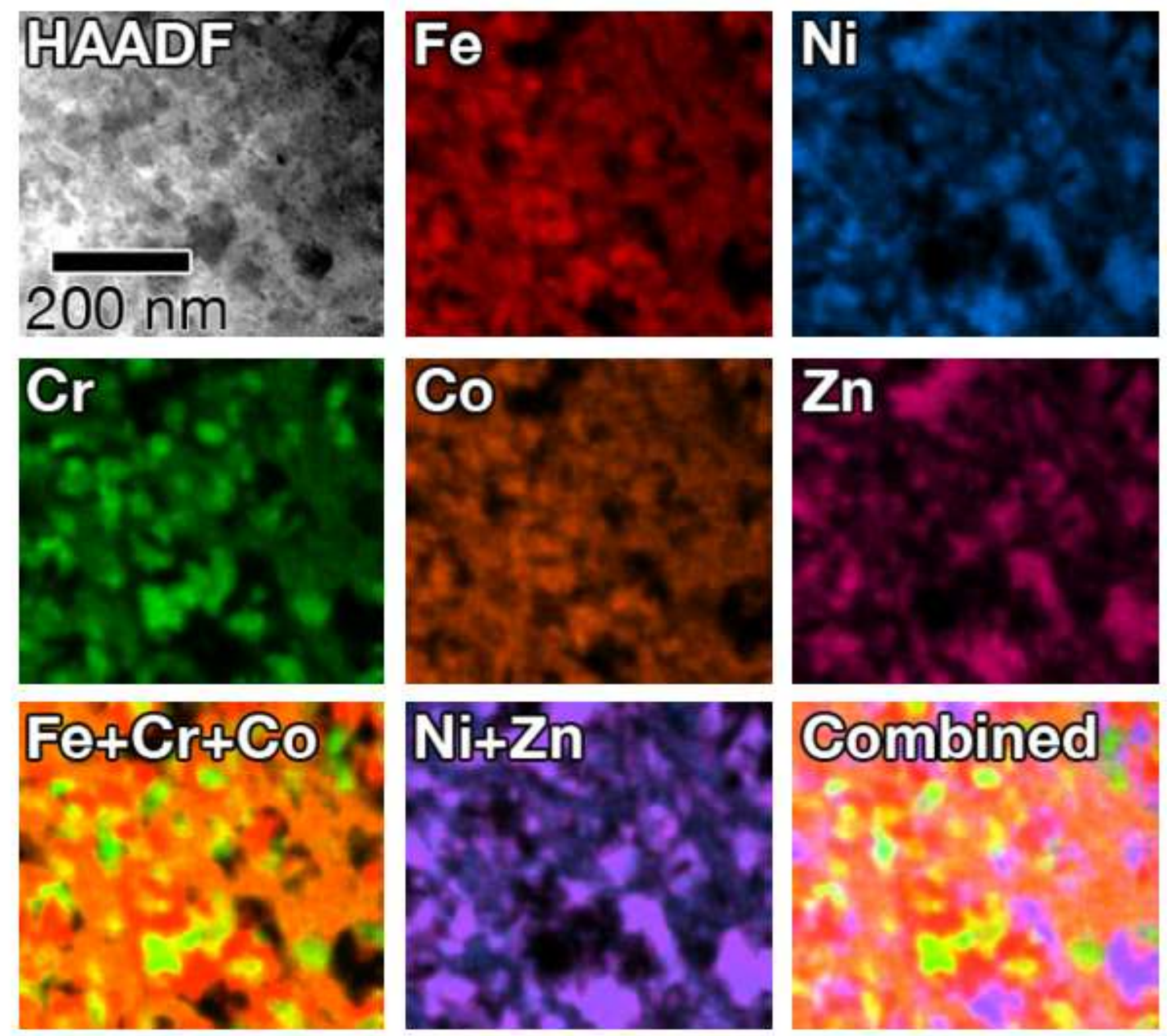

FetCrtGo

Nitzin

Gombined
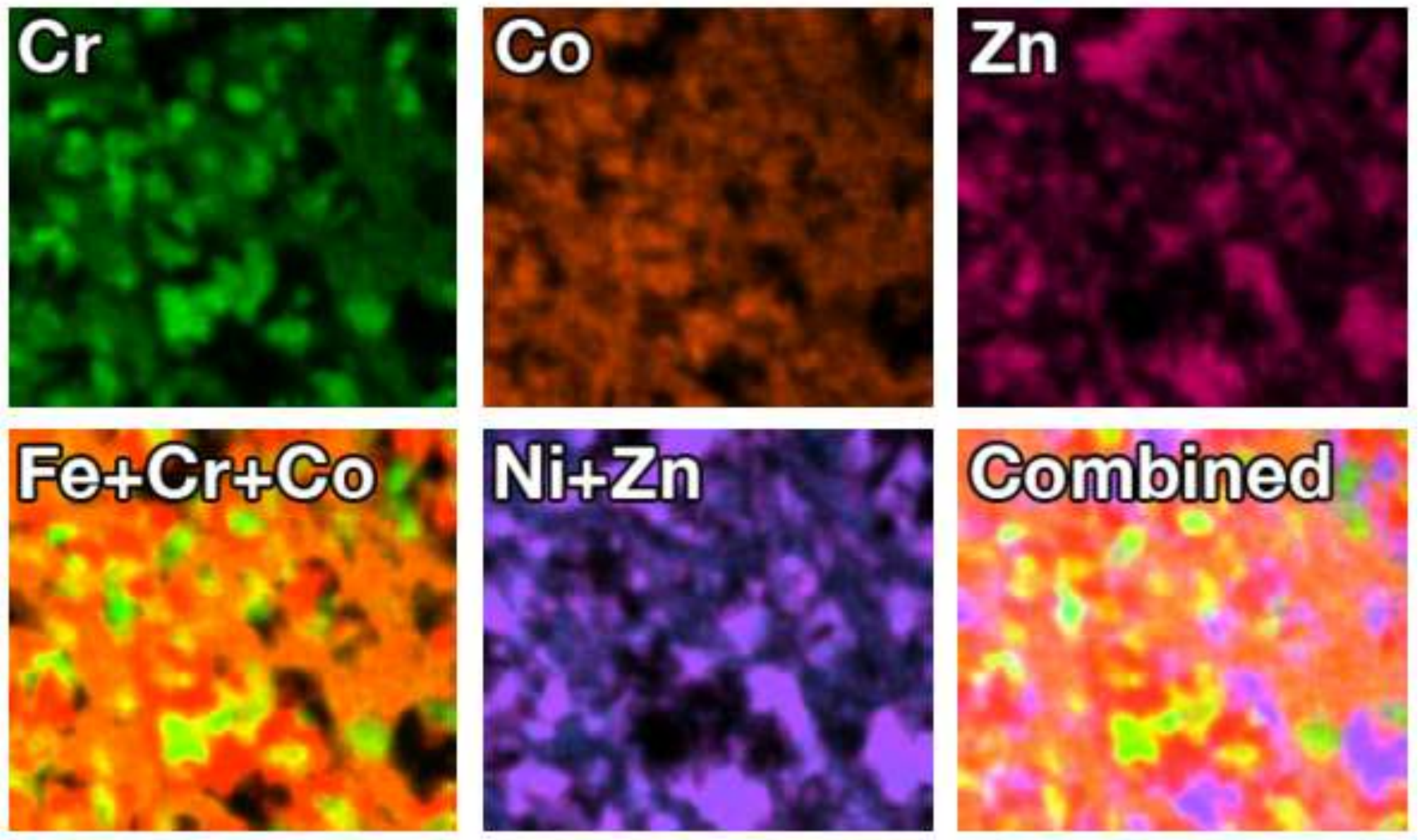


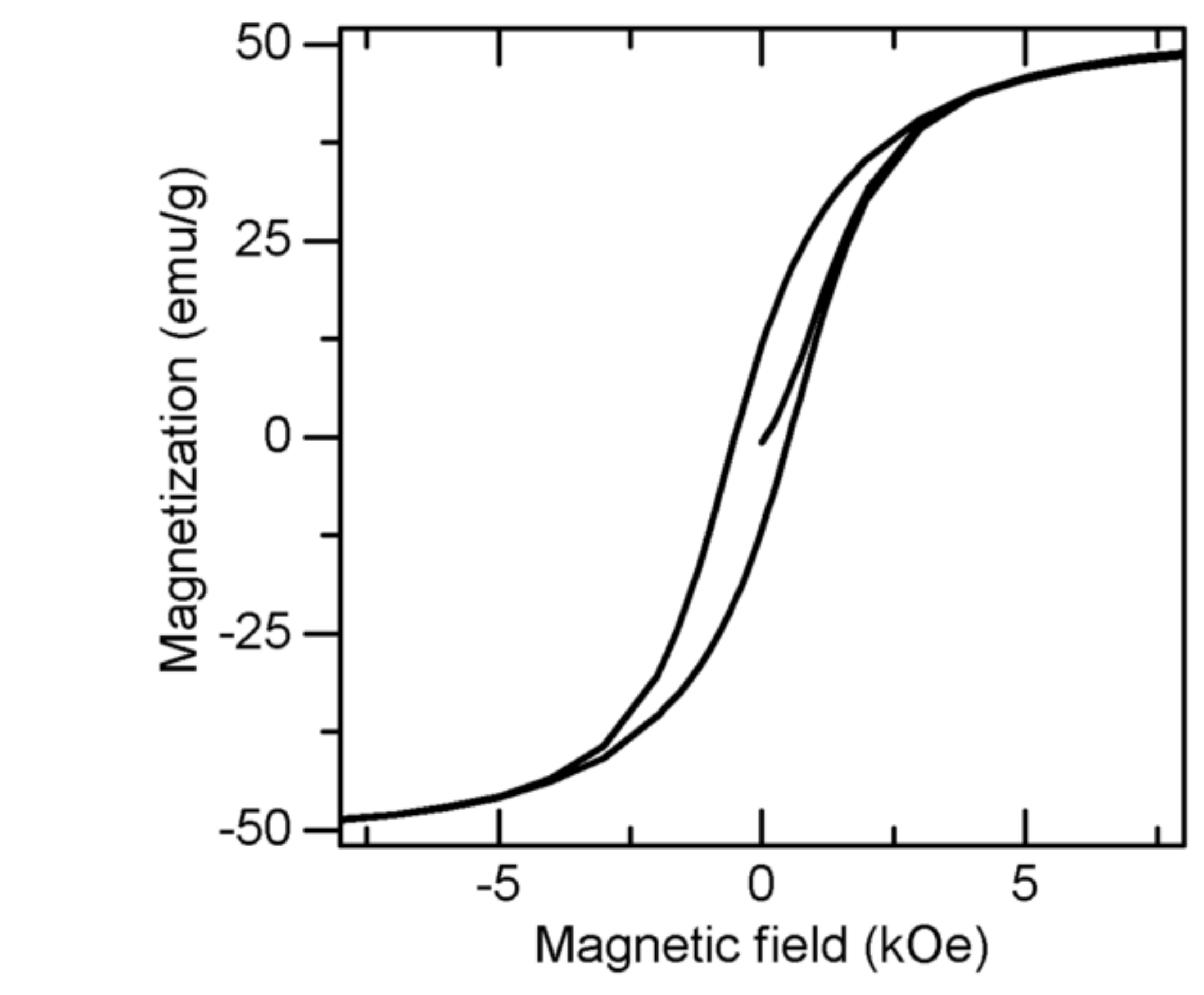

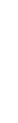




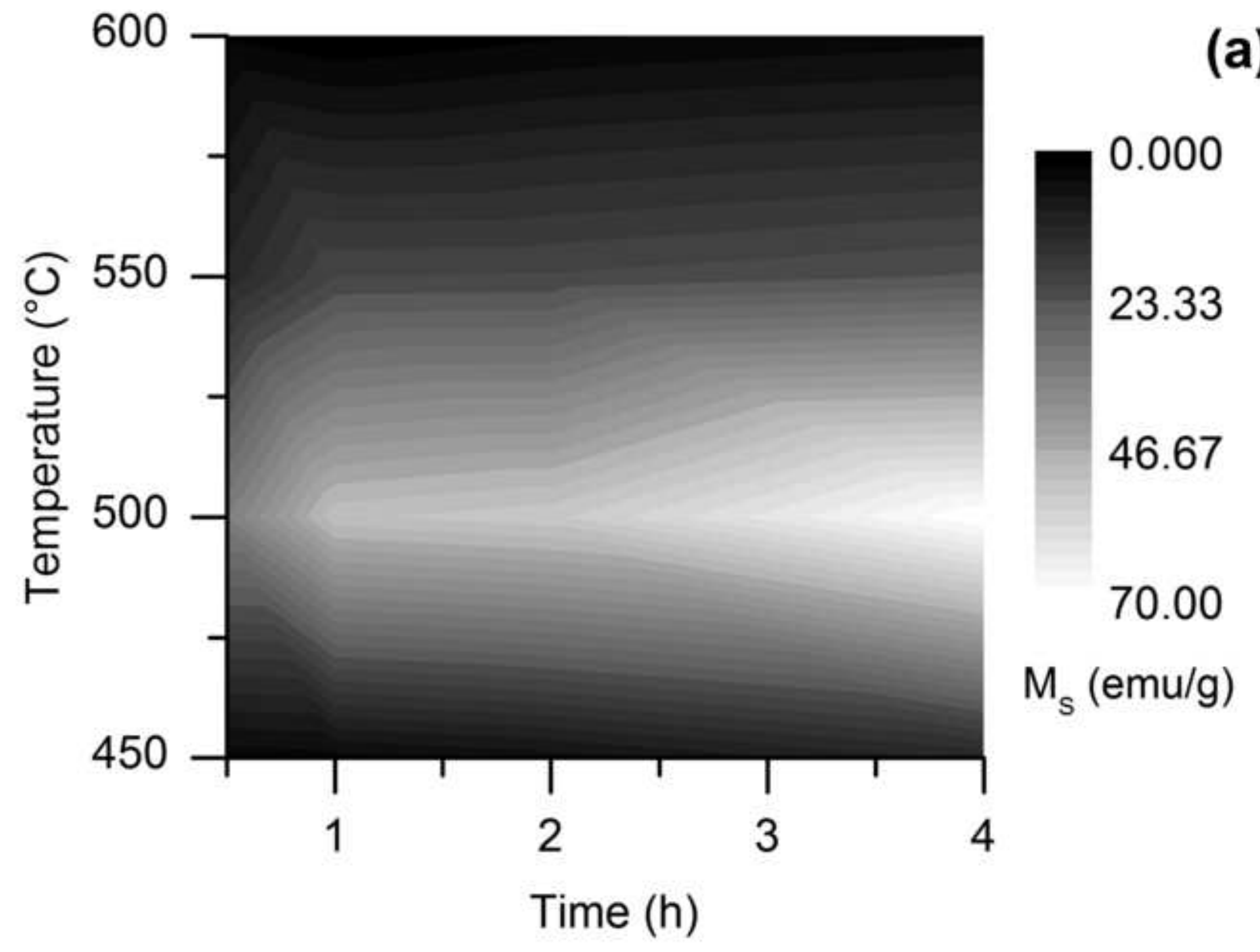




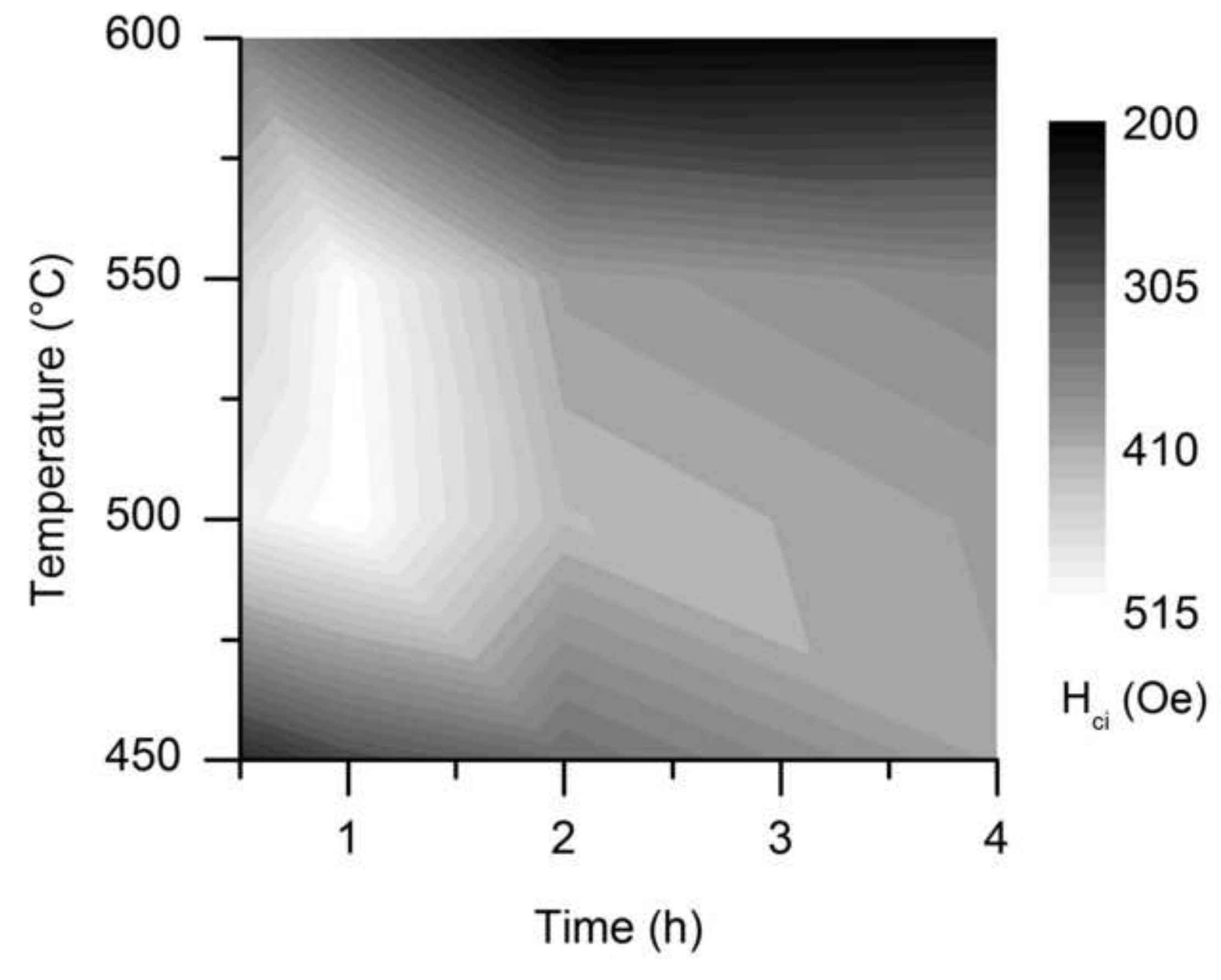

Time (h)

(b) 


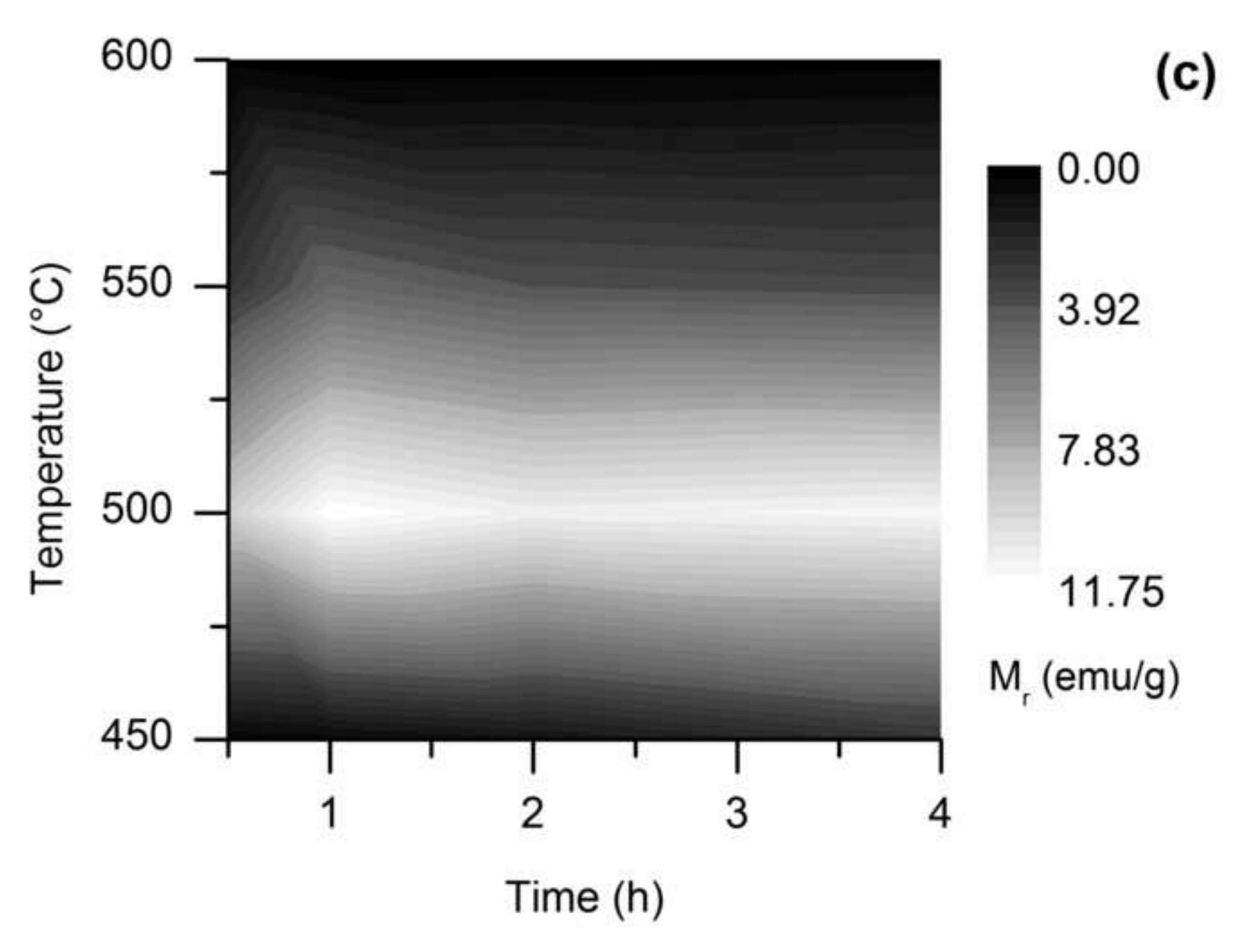




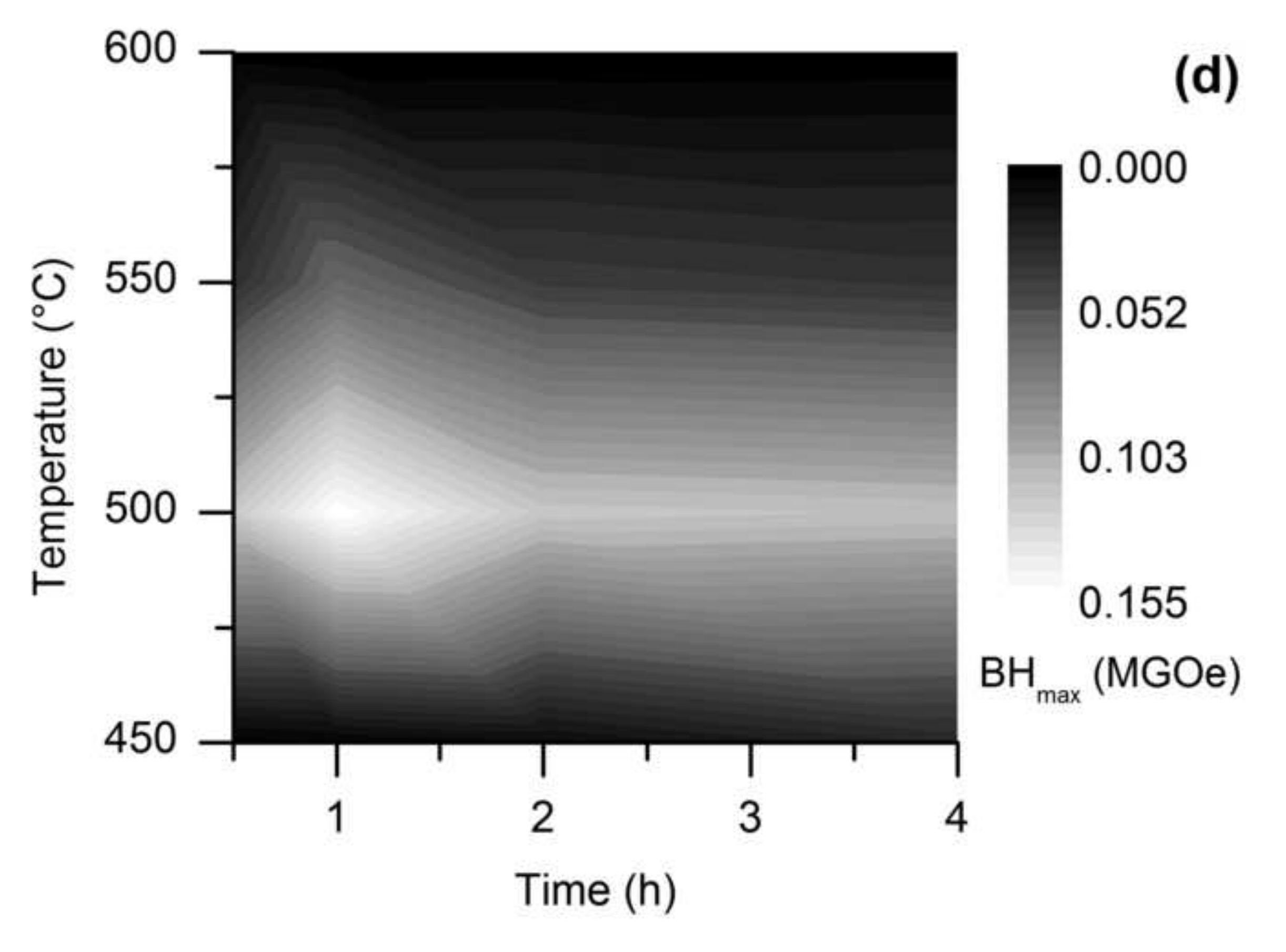

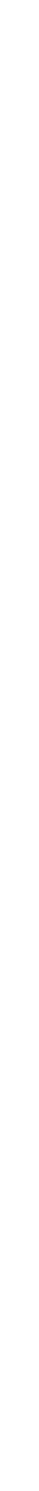

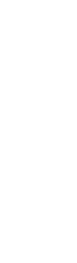

更

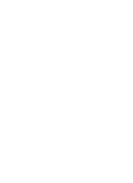

.
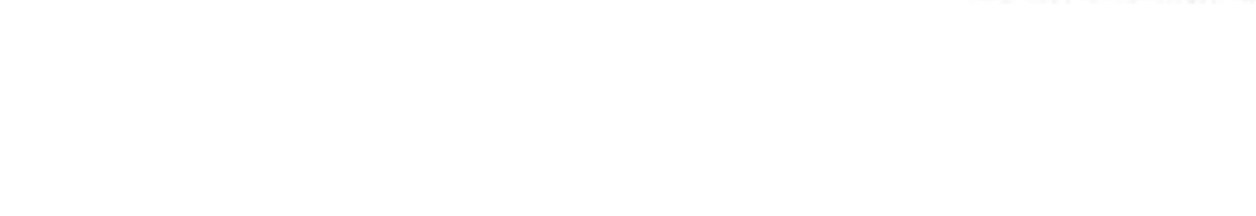

.

